\title{
Les drogues dures, poison ou répit ?
}

\author{
M.-F. Bacqué · S. Dauchy (Présidente de la SFfPO) \\ (C) Lavoisier SAS 2020
}

\section{Une crise des opiö̈des importée des États-Unis}

La peur de la douleur occupe une part importante dans les représentations négatives des cancers. La sédation de la douleur est donc l'une des demandes majeures des patients comme des soignants. Si les tentatives d'antalgie passent par des thérapeutiques médicamenteuses et non médicamenteuses, un signal d'alarme est tiré depuis les années 2000 aux États-Unis. La « crise des opioïdes » a fait plus de 50000 victimes américaines de 35 à 55 ans par overdose d'opioïdes antalgiques comme l'oxycodone.

En France, l'Agence nationale de sécurité du médicament (ANSM) a montré en février 2019 une augmentation de $150 \%$ des prescriptions d'opioïdes entre 2006 et 2017 [1]. Ces produits comme le tramadol, la codéine (tous deux antalgiques de niveau II), la morphine et le fentanyl (tous deux antalgiques de palier III, comme l'oxycodone) sont dorénavant moins tabous et même largement utilisés en France. En quinze ans, la mortalité liée à la consommation d'opioïdes en France a augmenté de $146 \%$, et la dépendance touche principalement les femmes (elles forment $54 \%$ des consommateurs de tramadol).

Les risques de dépendance et ceux du mésusage sont bien exposés par Khalida Berkane, addictologue de l'unité de psycho-oncologie et Sophie Laurent, algologue du Centre d'étude et de traitement de la douleur (CETD), toutes deux à l'Institut Gustave-Roussy. Elles nous présentent quatre cas de patient(es) qui montrent combien la multidisciplinarité des équipes du CETD est indispensable. L'enchevêtrement des différents facteurs qui président à la douleur sévère de certains cancers et des séquelles des traitements est dense en effet. Le mésusage des antalgiques et les vulnérabilités aux addictions liées à des facteurs génétiques ou à des facteurs personnels (comportementaux ou psychiatriques) s'additionnent aux autres surconsommations de toxiques ou de psy-

\footnotetext{
M.-F. Bacqué $(\bowtie)$

UR 3071, université de Strasbourg,

F-67000 Strasbourg, France

e-mail : mfbacque@club-internet.fr

S. Dauchy

SFPPO, F-75005 Paris, France
}

chotropes. La dépendance aux opioïdes et son mésusage entraînent des addictions qui peuvent être évaluées grâce à l'Opioid Risk Tool (ORT) et au Prescription Opioid Misuse Index (POMI).

Heureusement, des facteurs de protection permettent aux oncologues prescripteurs d'opioïdes de redonner de la responsabilité aux patients et de l'autonomie face aux risques addictifs. L'information des patients sur les risques du mésusage, le repérage de ses signes, l'adoption d'une prévention de ces risques, et enfin le soutien par les entourages familiaux, sociaux ou professionnels, améliorent considérablement la prise en soins. Ainsi, les effets de la médiatisation de la crise des opioïdes américaine, qui avaient conduit certains patients traités pour leur cancer à demander l'interruption ou l'arrêt de leur traitement morphinique, ont pu être limités.

\section{Les effets du cannabis insuffisamment documentés}

Les choses changent également du côté du cannabis : l'Assemblée nationale a voté en juillet 2019 l'ouverture d'une mission parlementaire dédiée au lancement d'une expérimentation du cannabis en thérapeutique pour une liste d'indications restreintes, dont certaines en oncologie.

La France est la championne d'Europe de la consommation de cannabis chez les 18-64 ans. Nous avions déjà recensé un ouvrage (2) qui faisait le point sur les usages du cannabis médicinal et bien relevé les différences entre Sativa et Indica ainsi que leur teneur en delta-9-tétrahydrocannabinol (THC) et en cannabidiol ou CBD. Les études citées par Natacha Naoun qui signe cet article sur cannabis et cancer sont encore incertaines quant à ses effets. Les douleurs chroniques, les douleurs neuropathiques, les nausées, les vomissements ont encore besoin de recherches systématisées pour apporter un résultat valide. La consommation de cannabis sous sa forme inhalée est un facteur de risque indépendant de tumeur germinale testiculaire, notamment non séminomateuse chez les jeunes hommes. Et pour le risque de cancer du poumon, la fumée issue du cannabis contient les mêmes toxiques que la fumée de cigarette : monoxyde de carbone, ammoniaque, acétaldéhyde, formaldéhyde, acroléine, phénols, nitrosamines, 
hydrocarbones polycycliques aromatiques. Quelques indications de traitement de la douleur peuvent se faire au cas par cas. Elles seront cependant modulées par des effets secondaires digestifs (syndrome cannabinoïde), psychotropes et un risque d'addiction en cas de consommation chronique.

\section{Pourquoi l'arrêt du tabac est-il peu fréquent après un diagnostic de cancer des voies aérodigestives supérieures?}

La comparaison des échanges en consultation de chirurgiens ORL dans un CLCC de la région Auvergne-Rhône-Alpes et de 27 patients atteints d'un cancer épidermoïde des voies aérodigestives supérieures montre que le fait de ne pas parler des liens entre le tabagisme et le cancer renforce le refus et même le déni du nécessaire sevrage. L'équipe de Mélanie Brossier de l'Inserm et celle de Sophie Deneuve du Centre Léon-Bérard de Lyon montrent, dans leur analyse thématique des discours des différents protagonistes de la consultation, que la cigarette occupe toujours dans les milieux les plus touchés par la précarité ou les difficultés économiques une place à part. Le tabac favorise les relations sociales entre fumeurs, il est systématiquement associé à la détente. La cigarette n'a pas qu'un usage social, elle aide aussi psychologiquement en fournissant un appui, un support et même un indice mnésique des histoires de vie des fumeurs. Les mots du chirurgien et les propositions d'accompagnement thérapeutique doivent donc être choisis à partir d'une évaluation qui permettrait d'identifier la composante relationnelle de l'alcoolo-tabagisme (les deux consommations vont souvent de pair). L'arrêt total du tabac ne peut pas être prescrit trop brutalement, mais ne pas structurer un dialogue sur l'arrêt entérine le déni du patient. Plus de souplesse et plus d'alliance entre les différentes équipes médicales (oncologie, addictologie) permettront aux fumeurs atteints de cancer de modifier une trajectoire de vie dans laquelle la pause cigarette, la cigarette, témoin de l'identification entre amis, celle dont la toxicité est contredite par le conformisme ambiant, forment une attache parfois indéfectible. En outre, les chirurgiens ne peuvent pas réduire leur action à leur bilan diagnostique et préopératoire. En montrant leur intérêt et leur participation à un programme d'accompagnement, ils pourront progressivement convaincre leur patient d'un sevrage qui lui donne toutes ses chances thérapeutiques.

\section{Un programme « star » pour limiter les addictions}

Le Département des soins de support et celui de la prévention de l'Institut régional du cancer de Montpellier proposent de profiter d'une de ces périodes pédagogiques (teachable moments) pour mettre en place un programme de « Sevrage Tabac-Alcool et Réduction des risques » (STAR). Partant des besoins de patients atteints de cancer, ce programme a été testé auprès de 30 patients par Anne Stoebner-Delbarre, addictologue et ses collègues chercheurs et infirmiers. Cette équipe pluriprofessionnelle s'est fondée sur les recommandations en addictologie-tabacologie et a appliqué les grands concepts de l'éducation thérapeutique pour soutenir le changement de comportement des patients. Les résultats sont encourageants et seront évalués en 2022. Le programme STAR s'adresse aux patients motivés par l'arrêt du tabac, mais il va plus loin que l'interaction éducateur/patient, il prône le partage d'information avec les autres professionnels et les participants grâce aux informations éducatives disponibles, avec accord des participants, dans leur dossier médical informatisé.

\section{Comment comprendre les addictions sous l'angle neurocognitif ?}

Xavier Noël, du Fonds national de la recherche scientifique (FRS/FNRS), professeur à l'Université libre de Bruxelles, nous présente les principaux modèles neurocognitifs actuels pour comprendre les mécanismes complexes des addictions. La plupart des millions de consommateurs de drogues, de jeux de hasard et d'argent et maintenant de jeux vidéo, ne parviennent pas à comprendre que leur désir intense de consommer, leur difficulté de reprise de contrôle, les phénomènes de tolérance et de dépendance ainsi que l'abandon progressif d'autres sources de plaisir et d'intérêt sont d'origine cérébrale. La recherche en neurosciences cognitives permet aujourd'hui d'éclairer ces phénomènes. Les différents conditionnements de la vie, la bascule entre recherche du plaisir et évitement du déplaisir, la répétition des expériences addictives, maintiennent le consommateur dans son addiction, pire créent un phénomène de résistance à l'extinction du comportement. Ainsi, même la modification de l'expérience ressentie, comme le remplacement du pop-corn frais par du pop-corn rance, pour les personnes qui ne peuvent se priver de manger du pop-corn au cinéma, sera sans effet. Ce qui compte c'est la satisfaction d'une séquence qui répète les conditions initiales, même si le goût de l'expérience première a depuis longtemps disparu.

Les propositions thérapeutiques s'appuyant sur ces dysfonctionnements neurocognitifs sont parfaitement illustrés par Xavier Noël, dans le respect de la complexité des mécanismes de l'addiction, à la croisée des facteurs psychologiques et sociétaux.

Ce dossier « Cancers et addictions » fait donc le point sur un thème délicat, dont l'importance et la complexité ne peuvent être ignorées des psychologues et des psychiatres exerçant en cancérologie. L'alccol et le tabac sont de puissants 
cancérogènes, et les mécanismes comme les effets de leur consommation impliquent le psychisme de nos patients. Les douleurs des cancers sont traitées par des molécules à potentiel addictogène : nous sommes tous concernés par ce risque pour les patients que nous suivons, dont la qualité de vie va souvent être conditionnée par le recours à ces antalgiques opiacés. Les oncologues doivent pouvoir maitriser leurs effets. Les psychologues et les psychiatres ont besoin d'être parfaitement documentés sur leur utilisation notamment pour limiter les représentations fausses et dans la collaboration avec les prescripteurs, oncologues, algologues ou psychiatres pour prévenir le mésusage et soutenir les patients douloureux. Le parcours semé d'embûches des patients dépendants atteints de cancer peut maintenant être mieux accompagné grâce à cette mise au point scientifique et thérapeutique.

\section{Référence}

1. Bacqué MF (2016) Cannabis médicinal. Mieux se soigner avec la marijuana. De Michael Backes. Psycho-Oncol 10:227-30 